\section{Investigations on Nano- and Pico-Second Laser Based Annealing Combined Texturing of Amorphous Silicon Thin Films for Photovoltaic Applications*}

\author{
Gomathinayagam AMUTHA**, Iyamperumal Anand PALANI***, \\ Nilesh Jayantilal VASA**, Makaram SINGAPERUMAL**** \\ and Tatsuo OKADA***** \\ **Department of Engineering Design, Indian Institute of Technology Madras, Chennai 600036, India \\ ${ }^{* * *}$ Mechanical Engineering Discipline, Indian Institute of Technology Indore, Indore 452017, India \\ ${ }^{* * * *}$ Department of Mechanical Engineering, Indian Institute of Technology Madras, \\ Chennai 600036, India \\ ${ }^{* * * * *}$ Graduate School of Information Science and Electrical Engineering, Kyushu University, \\ Fukuoka 8190395, Japan \\ E-mail: gamutha@iitm.ac.in, njvasa@iitm.ac.in, palaniia@iiti.ac.in
}

\begin{abstract}
Influence of a nano- (ns) and a pico-second (ps) laser pulse in annealing and texturing of amorphous-silicon (a-Si) films on crystalline silicon substrates by pulsed $\mathrm{Nd}^{3+}:$ YAG lasers is investigated. In theoretical studies, the thermal model was considered for the ns-laser annealing and the plasma model with an appropriate energy relaxation time was used for the ps-laser annealing, as both the processes are different on a time-scale. Crystallization depth was estimated at different wavelengths, and interface temperature between a-Si films and c-Si substrates was compared. Modeling results shows that with a ns-laser annealing at longer wavelength $(1064 \mathrm{~nm})$ has a weak absorption and therefore higher laser fluence is required to raise the film temperature to a required level and it was in agreement with the experimental results. Also the estimated heat diffusion region at the a-Si/substrate interface was significant due to the ns-order pulse-width. Whereas, in the case of a ps-laser $(1064 \mathrm{~nm})$, it was possible to anneal the a-Si film with a lower laser fluence. In addition, the estimated heat diffusion length at the a-Si film/substrate interface was lower than the ns-laser assisted treatment.
\end{abstract}

Key words: Nano-Second Laser, Pico-Second Laser, Annealing, Texturing, Amorphous Silicon, Polycrystalline Silicon

\section{Introduction}

Semiconductors such as crystalline silicon play a major role in the application of MEMS devices and photovoltaic application such as solar cells. However the resulting cost is high and overall size is limited. In substitute of these, low cost amorphous silicon films can be considered for photovoltaic applications. However the photovoltaic efficiency is limited due to absence of crystalline structure. Efficiency can be improved through laser annealing and texturing technique. In the laser annealing technique, a high intensity short
${ }^{*}$ Received 17 Sep., 2012 (No. 12-0370) [DOI: 10.1299/jmmp.7.206]

Copyright $\odot 2013$ by JSME 
duration laser beam is focused on to the amorphous silicon (a-Si) film, which induces crystallization and produce poly-crystalline silicon films by fast heating and cooling ${ }^{(1,2,3)}$. An approach based on a solid-state laser, such as a pulsed $\mathrm{Nd}^{3+}$ :YAG $\left(\mathrm{Nd}^{3+}\right.$-ion doped yttrium aluminum garnet) laser, can be considered for annealing of a-Si film on a suitable substrate $^{(4)}$

To induce texturization the laser spots were overlapped to $90 \%$ of its diameter by translating the sample stage. This was expected to lead to a high temperature gradient in the interface region resulting in a convective motion know as the Marangoni effect. On cooling, due to the decrease in temperature and the surface gradient, the convective motion originating due to the Marangoni effect will be damped, resulting in formation of roughness pattern. It produces a textured surface on the silicon surfaces so as to decrease the reflection from the surface and improve the light trapping efficiency ${ }^{(5,6)}$. However formation of a heat affected zone with the nanosecond laser (ns-laser) pulse influences the film characteristics. These can be overcome by using an ultra-short laser pulse processing technique. In this paper an attempt has been made for the first time by using a pico-second laser (ps-laser) for laser based annealing and subsequent texturing.

The laser fluence, pulse width and pulse repetition rate are crucial parameters in facilitating the type of mechanism the process adopts for different materials. Several researchers have reported about the influence of these parameters on laser assisted processing of different materials. However, the interaction mechanism of semiconductor material with a pulsed laser is not clear $^{(7)}$. For example, a nanosecond pulse incident on a amorphous silicon film results in the formation of a thin molten layer and crystallization occurs while cooling, While that with a ps-laser pulse incidence with same energy produce high density plasma results in an ablation of the film ${ }^{(8,9)}$. Also, as the heat affected zone (HAZ) depends on pulse width, and heat affected area is expected to reduce with the ps-laser annealing ${ }^{(10,11)}$.

In this paper, the process of laser interaction with amorphous silicon and the temperature evolution of the surface and the substrate with nano- (ns) and pico-second (ps) laser pulse are theoretically investigated. In ns-laser assisted annealing, the influence of the wavelengths $355 \mathrm{~nm}, 532 \mathrm{~nm}$ and $1064 \mathrm{~nm}$ is investigated. But in ps-laser assisted annealing, the effect by the wave length of $1064 \mathrm{~nm}$ is only investigated because, at $355 \mathrm{~nm}$ and $532 \mathrm{~nm}$, with ns-laser annealing, the absorption coefficient value is large and with moderate laser fluence itself, crystallization temperature is achieved at the surface without raising the substrate temperature. Hence with $355 \mathrm{~nm}$ and $532 \mathrm{~nm}$ wavelengths, ns-laser annealing provides appropriate annealing ${ }^{(5)}$. Whereas with ps-laser annealing, as the absorption coefficient value at $355 \mathrm{~nm}\left(1 \times 10^{8} \mathrm{~m}^{-1}\right)$ and $532 \mathrm{~nm}\left(1 \times 10^{7} \mathrm{~m}^{-1}\right)$ wavelengths is larger than that of at $1064 \mathrm{~nm}\left(1 \times 10^{6} \mathrm{~m}^{-1}\right)$ wavelength, amorphous-silicon film is expected to be ablated with a lesser fluence value due to the strong absorption and a high density plasma effect as a result of free-carrier absorption. The depth of penetration is also expected to be lesser resulting in a thin annealed layer. Hence, fundamental wavelength of $1064 \mathrm{~nm}$ was selected for the ps-laser annealing experiments. Experimentally, laser annealing and texturing is done on a amorphous silicon film with the ns- and the ps-laser at the wave length of $1064 \mathrm{~nm}$. The treated samples are analyzed by a scanning electron microscope (SEM) and Raman Spectroscopy to investigate the surface morphology. 


\section{Nano-second and pico-second lasers interaction with matter}

\subsection{Nano-second pulsed laser processing (Macroscopic Modeling)}

In the case of a ns-laser pulse, the pulse duration (few ns order) is larger than the time required for transfer of energy from electrons to the lattice $\left(10^{-12} \mathrm{~s}\right)$. When a ns-laser beam is focused on to the film, the incident photons are absorbed instantaneously and a macroscopic model of heat absorption can be considered ${ }^{(12)}$. Properties, such as thermal conductivity, specific heat and absorption coefficient completely determine the thermal state of the film and the substrate. The amount of energy absorbed by the film goes on decreasing with the depth given by the Beer-Lambert's law. The absorbed energy diffuses, in accordance with the thermal gradient and the thermal conductivity by collisions and rising the temperature of the surface. The distances to which the heat diffuses are very short and the large dimensions of the laser beam compared to the melt depth, essentially limit the thermal gradients parallel to the interface to many orders of magnitude lesser than the gradients present perpendicular to the interface. Thereby it is essentially a one dimensional problem. The laser pulse processing may well be described using the thermal model which is based on one-dimensional heat flow given by ${ }^{(12)}$,

$c_{S}(T) \rho_{S}(T) \frac{\partial T(x, t)}{\partial t}=\frac{\partial}{\partial x}\left(\kappa(T) \frac{\partial T(x, t)}{\partial x}\right)+S(x, t)$.

Where $T(x, t)$ is the temperature distribution, $C_{p}(T)$ is the specific heat, $\rho_{s}(\mathrm{~T})$ is the density, $k(T)$ is the thermal conductivity.

The source term given by $S(x, t)$ is proportional to the energy absorbed by the surface layers given by:

$Q(x, t)=Q_{\text {pulse }}(t)(1-R(T)) \exp [-\alpha(T) x]$,

where $R(T)$ is the reflectivity, $\alpha(T)$ is the absorption coefficient and $Q_{\text {pulse }}(t)$ is laser pulse energy. The energy loss due to the Planck radiation, surface evaporation at the melting point and heat convection to the surroundings is very small in comparison with the laser beam energy and therefore is not included into the source term $\left(Q_{\text {loss }}<10^{-3} Q_{\text {pulse }}\right)^{(2)}$.

\subsection{Pico-second pulsed laser processing (Microscopic modeling)}

In the case of ps-laser annealing, very high intensity short duration laser pulse creates dense, hot electron-hole plasma which results high concentration of carriers with high carrier temperature ${ }^{(13)}$. As the energy relaxation time of carriers is proportional to square of the carrier concentration, carriers take longer time to relax their energy to the lattice, so the lattice cannot get same temperature of the carrier. Hence the thermal model cannot be used. An alternate theory for annealing mechanism based on the plasma model with appropriate relaxation time for energy transfer from carrier to the lattice can be considered $^{(14)}$.

A schematic diagram of the model is given in Fig 1. The equations for the lattice temperature $T$, the carrier concentration $p(x, t)$, carrier energy $E_{c}(x, t)$ and the laser intensity, $I(x, t)$, are given by ${ }^{(14)}$,

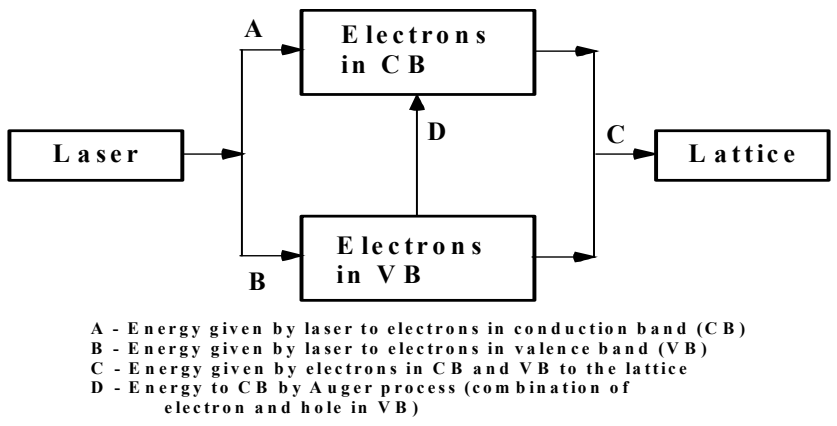

Fig.1. Schematic of microscopic thermal process 


$$
\begin{aligned}
& \rho C p(T) \frac{\partial T(x, t)}{\partial t}=\frac{\partial}{\partial x}\left(k(T) \frac{\partial T(x, t)}{\partial x}\right)+\left(\frac{E_{c}(x, t)-3 p(x, t) k_{b} T(x, t)}{\tau_{\varepsilon}}\right) \\
& E_{\text {carr }}=3 k_{b} T_{e} \\
& \frac{\partial p(x, t)}{\partial t}=\frac{\partial}{\partial x}\left(D_{a} \frac{\partial p(x, t)}{\partial x}\right)+\frac{\partial}{\partial x}\left(\mu_{a} p(x, t) \frac{\partial\left(E_{g} / 2\right)}{\partial x}\right)+\frac{I(x, t) a_{l}}{E_{\text {Phot }}}-\frac{\left(p(x, t)-p_{0}\right)}{\tau_{p}} \\
& \frac{\partial E_{c}(x, t)}{\partial t}=\frac{\partial}{\partial x}\left(\frac{E_{c}(x, t)}{p(x, t)} D_{\alpha} \frac{\partial p(x, t)}{\partial x}\right)+\left(\frac{E_{p h-} E_{g}}{E_{p h}} \alpha_{L}+\alpha_{F C A}\right)+E_{g}\left(\frac{\left(p(x, t)-p_{0}\right)}{\tau_{p}}\right) \\
& -\left(\frac{E_{c}-3 p(x, t) k_{b} T(x, t)}{\tau_{\varepsilon}}\right) \\
& I(x, t)=I o \exp \left(\alpha_{L}+\alpha_{F C A}\right) x
\end{aligned}
$$

Where $\kappa(T)$ is the thermal conductivity, $\rho$ density and $C_{p}(T)$ the specific heat of a-Si. $E_{p h}$ is the photon energy and $E_{g}$ is the band gap. $\tau_{p}$ is the lifetime, $D_{a}$ the ambipolar diffusivity, and $p_{o}$ is the equilibrium concentration of carriers, $\tau_{e}$ is the energy relaxation time, $\mu$ is the ambipolar mobility, $\alpha_{L}$ is lattice absorption coefficient, $\alpha_{F C A}$ is the free carrier absorption coefficient.

In Eq. (5), the first term on the right represents diffusion of energy by carrier diffusion, the second term describes the effect of absorption, the third term is the energy added to the carrier system by Auger recombination and the fourth term is the emission rate from the carrier system to the lattice. Above equations were considered in the simulation.

\section{Experiment}

Experimental studies were performed using a ns-pulsed $\mathrm{Nd}^{3+}$ :YAG laser and a ps-pulsed $\mathrm{Nd}^{3+}$ :YAG laser. Table 1 describes laser parameters used in experimental studies. N-type crystalline silicon (c-Si) substrates $(500 \mu \mathrm{m})$ with amorphous silicon film (p-type, thickness $400 \mathrm{~nm}$ ) deposited using PECVD (Plasma Enhanced Chemical Vapor Deposition) process were used for the thin film annealing studies.

Table 1. Laser parameters

\begin{tabular}{|l|l|l|l|}
\hline \multicolumn{2}{|c|}{ ns-laser } & \multicolumn{2}{c|}{ ps-laser } \\
\hline Laser & $\mathrm{Nd}^{3+}:$ YAG & Laser & $\mathrm{Nd}^{3+}:$ YAG \\
\hline Wavelength & $1064 \mathrm{~nm}$ & Wavelength & $1064 \mathrm{~nm}$ \\
\hline Pulse Width & $9 \mathrm{~ns}$ & Pulse Width & $30 \mathrm{ps}$ \\
\hline Frequency & $10 \mathrm{~Hz}$ & Frequency & $10 \mathrm{~Hz}$ \\
\hline Diameter of the spot & $6 \mathrm{~mm}$ & Diameter of the spot & $6 \mathrm{~mm}$ \\
\hline
\end{tabular}




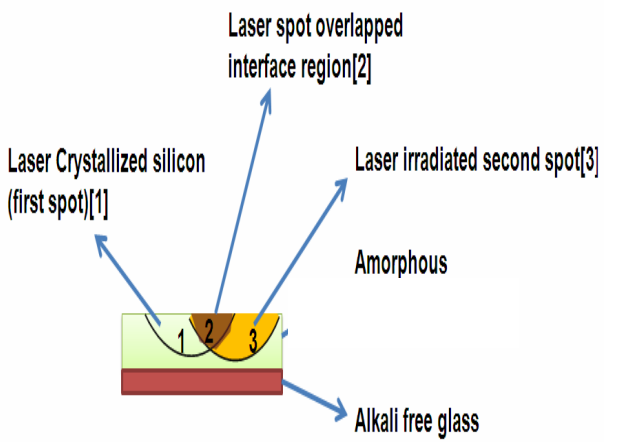

Fig.2. Regions of irradiated surface with laser spot overlap
The laser beam intensity profile was Gaussian and with a beam spot size of $6 \mathrm{~mm}$. Amorphous-Si thin film (400 nm) coated on a c-Si substrate was mounted on an X-Y translation-stage. To induce texturization, the first laser pulse was used to irradiate the a-Si film. After the phase transformation was completed the second laser spot was overlapped with the previous irradiated spot to $90 \%$ of its size.(Fig.2) The samples were treated

with different laser fluence values ranging from 100 to $400 \mathrm{~mJ} / \mathrm{cm}^{2}$ and from 900 to 1600 $\mathrm{mJ} / \mathrm{cm}^{2}$ for the ps-laser and the ns-laser treatment, respectively.

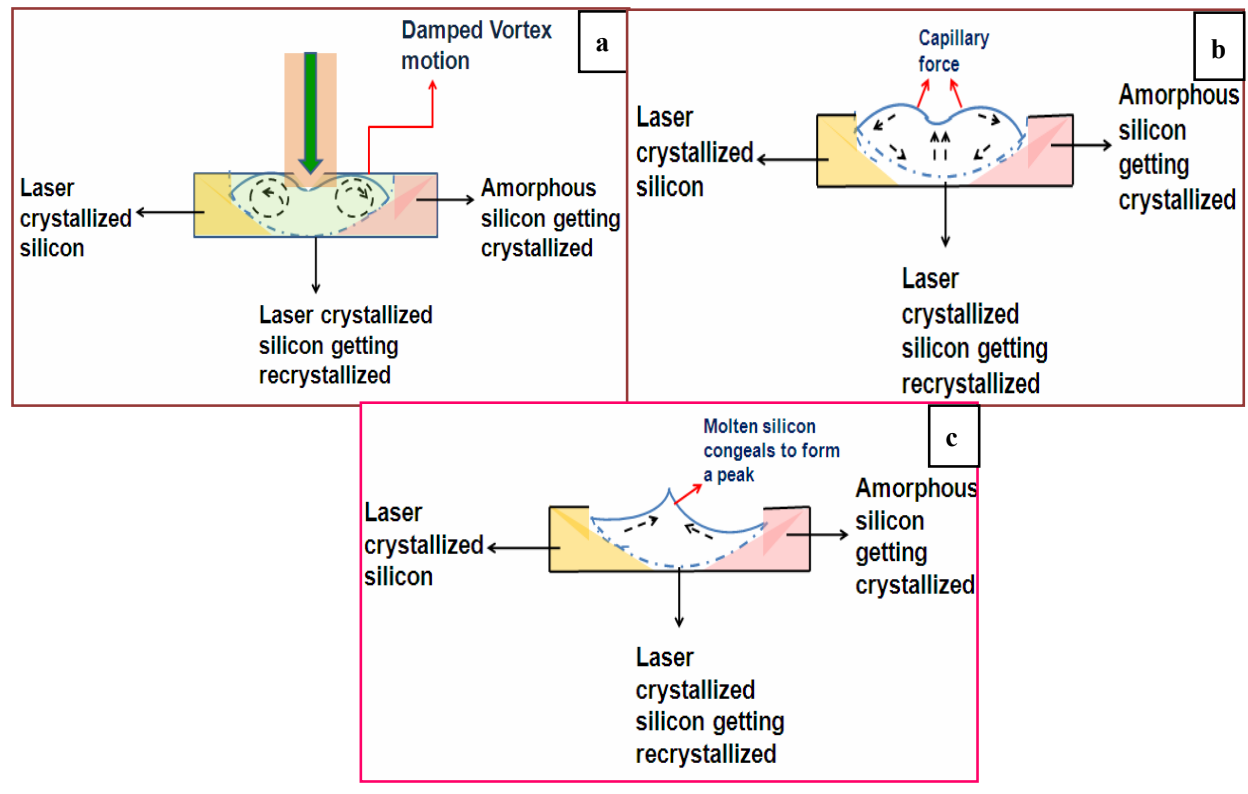

Fig. 3(a) laser annealing of a-Si

3(b) molten Si flow during laser annealing

3(c) molten Si flow during cooling

To understand the phenomena of laser assisted texturing with beam overlap, the Marangoni effect may be considered. The Marangoni convection occurs when the surface tension of an interface depends on the temperature distribution or the concentration of the species. In the case of temperature dependence, the Marangoni effect is called thermo capillary convection. Various stages of peak generation in the interface region are shown in Figs. 3 (a) to (c). The Marangoni effect is the mass transfer along an interface due to the surface tension gradient. Since a liquid with a high surface tension pulls more strongly on the surrounding liquid, the presence of gradient in the surface tension will naturally cause the liquid to flow away from the region of low surface tension. During the heating cycle, the Marangoni convection results in a vortex motion and liquid movement towards the region of low temperature as shown in Fig. 3 (a). On cooling, due to the decrease in temperature and the surface gradient, the convective motion originating from the Marangoni effect will be damped and the hydrodynamic motion will then evolve as shown in Fig. 3 (b). Capillary 
waves will be generated due to the surface tension. These capillary waves will exist in the liquefied region until the fused silicon congeals. During cooling, the amplitude of the surrounding surface will first decrease and transforming to its mirror image by passing a phase where there is practically a smooth surface. This may result in splashing of material along the liquid trench axis as shown in Fig. 3 (c). Hence the formation of peaks depends on the initial temperature of the liquid and the size of the liquid zone. This leads to the formation of a rough pattern at the interface ${ }^{(15)}$.

\section{Nano-second Vs Pico-second laser based annealing combined texturing 4.1 Surface morphological analysis}

Figure 4(a) shows the SEM image of a ns-annealed sample at1200 $\mathrm{mJ} / \mathrm{cm}^{2}$. In Fig.4(b), pinhole appearance with crystal defects are observed in the heat affected area and in Fig.4(c), microcrystal formation is observed in the crystalline region. Figure 5(a) shows the SEM image of the ps-laser annealed sample at a laser fluence of $195 \mathrm{~mJ} / \mathrm{cm}^{2}$, and here the heat affected zone is not observable. Figure 5(b) shows the microcrystal formation on the a-Si samples. Below $195 \mathrm{~mJ} / \mathrm{cm}^{2}$, no significant change was observed. With increase in laser fluence to $250 \mathrm{~mJ} / \mathrm{cm}^{2}$, ablation damage on a-Si film was observed.

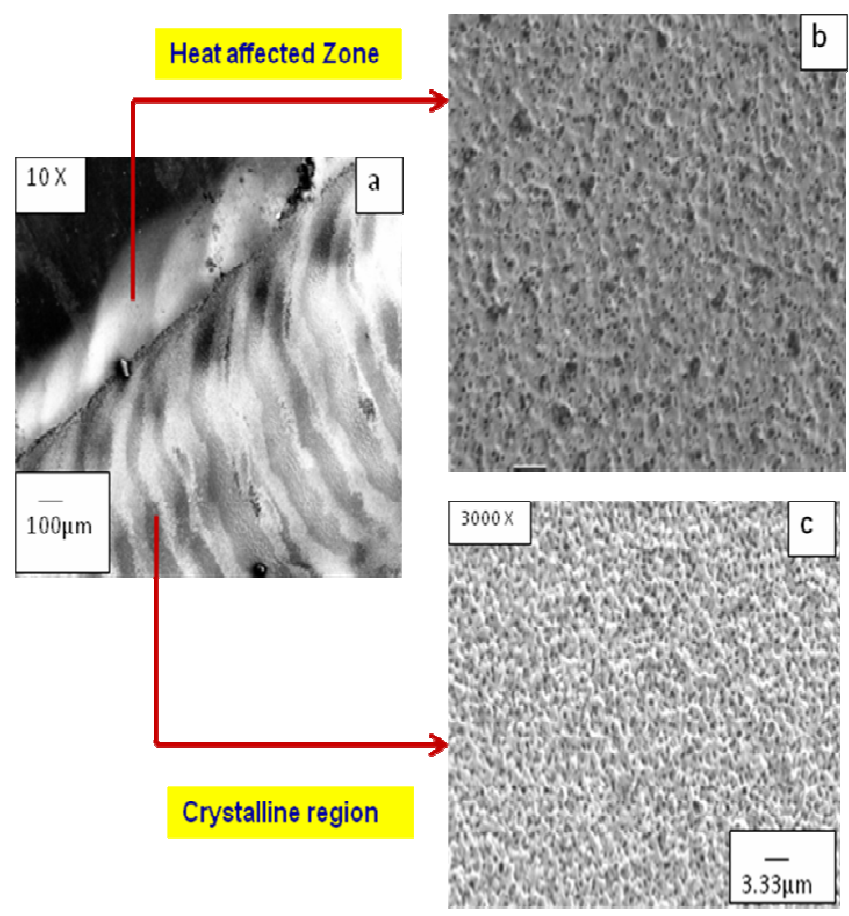

Fig.4 (a) ns-laser annealed sample, (b) Heat affected region, (c) Crystalline region

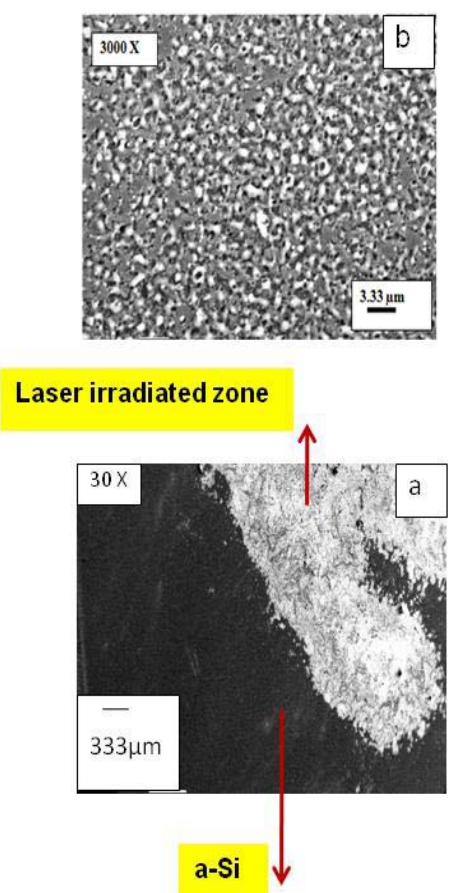

Fig.5 (a) ps-laser annealed sample, (b) crystalline region

\subsection{Annealing characteristics}

In Fig. 6, at $195 \mathrm{~mJ} / \mathrm{cm}^{2}$, a narrow-band peak at $519 \mathrm{~cm}^{-1}$ in the Raman spectrum was observed which was comparable with samples annealed with the ns-lased pulse at 1064 $\mathrm{nm}$. With the laser fluence of $250 \mathrm{~mJ} / \mathrm{cm}^{2}$, the peak shifted to $510 \mathrm{~cm}^{-1}$ which confirmed an ablation of the film. Figures 7(a) and (b) show resistance measurements for the ns- and ps-laser treated samples, respectively. As observed in Fig. 7(a), in the case of the ns-laser 
annealing, the film resistance decreased between the laser fluence of 900 and $1200 \mathrm{~mJ} / \mathrm{cm}^{2}$, and above the range, the resistance increased due to the film ablation. In the case of the ps-laser assisted annealing, with the laser fluence ranging from 100 to $195 \mathrm{~mJ} / \mathrm{cm}^{2}$, the film resistance values decreased which show the crystalline region and beyond this value, the film resistance increased due to the ablation. When the laser fluence value was increased beyond the process-window, the film was ablated and crystal characteristics of the substrate were also affected at a higher laser fluence value due to the heat diffusion into the substrate. This variation was also observed in the Raman spectra ${ }^{(4)}$. The damage caused to the crystalline region is the probable cause for an increase in the resistance value.

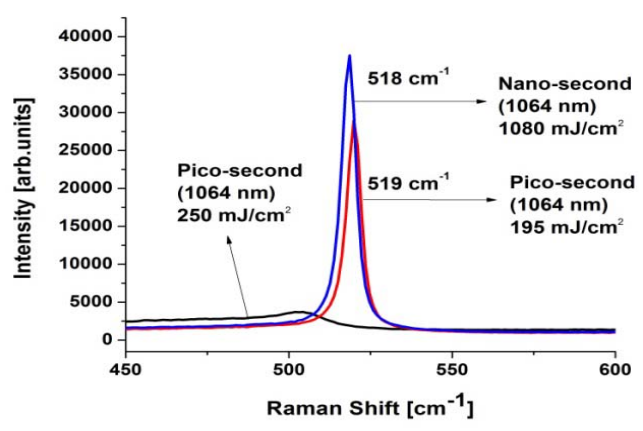

Fig.6. Raman Spectroscopic analysis

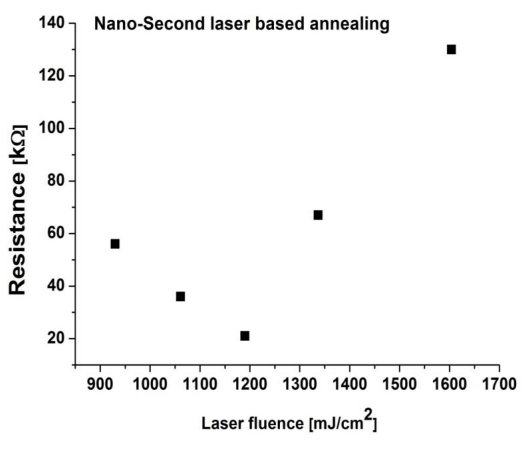

Fig.7(a) Resistance measurement in ns-laser annealed sample

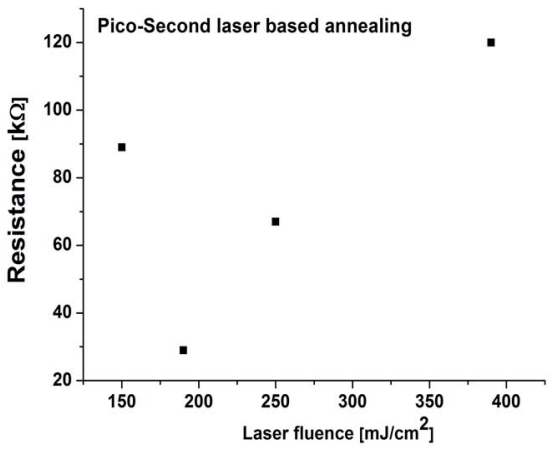

Fig.7(b) Resistance measurement in ps-laser annealed sample

\subsection{Texturization characteristics}

The heat affected zone regions have their own influence towards generation of peak. This mechanism was confirmed through the AFM analysis. Fig. 8 shows the AFM image of the textured peak by 30 ps laser pulse. Table 3 shows the RMS (root mean square of surface roughness) measurement at $90 \%$ overlap. The samples crystallized with the ps-laser showed an enhanced roughness parameter value, $\mathrm{Rq}$, as compared to the samples annealed with the ns- laser pulse. 


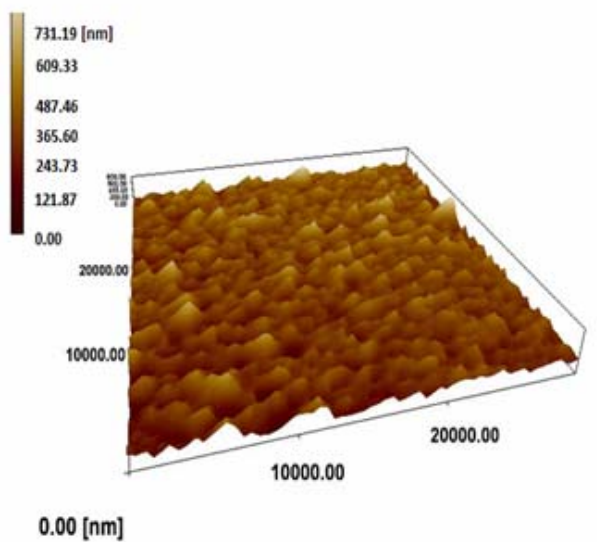

Fig \&. AFM image of nanotextured surface by $30 \mathrm{ps}$. $1064 \mathrm{~nm}$.
Table 3. Roughness $(\mathrm{Ra})$ measurement

\begin{tabular}{|l|l|l|}
\hline & $\begin{array}{l}\text { Laser Fluence } \\
{\left[\mathbf{m J} / \mathbf{c m}^{2}\right]}\end{array}$ & $\begin{array}{l}\mathbf{R q} \\
{[\mathbf{n m}]}\end{array}$ \\
\hline $\begin{array}{l}\text { Pico-Second } \\
\text { Laser (1064 } \\
\mathrm{nm})\end{array}$ & $\begin{array}{l}195 \\
\text { (Crystallization) }\end{array}$ & 570 \\
\hline & 250 (Ablation) & 260 \\
\hline $\begin{array}{l}\text { Nano-Second } \\
\text { Laser (1064 } \\
\text { nm) }\end{array}$ & $\begin{array}{l}1080 \\
\text { (Crystallization) }\end{array}$ & 150 \\
\hline & 1400 (Ablation) & 120 \\
\hline
\end{tabular}

\subsection{Theoretical analysis on crystallization depth}

In the a-Si film, crystallization temperature region is between $1073 \mathrm{~K}$ and $1573 \mathrm{~K}$. In the theoretical simulation, the laser fluence required to attain the crystallization without ablation was estimated. The ability of the material to absorb laser energy limits the depth to which crystallization can be attained. Crystallization depth also depends on other parameters such as thermal conductivity of the material, beam energy density (laser fluence), the laser pulse duration, and the laser wavelength. For a-Si film, the absorption depth increases as the wavelength increases from $355 \mathrm{~nm}$ to $1064 \mathrm{~nm}$. From Figs. 9(a) $9(\mathrm{c})$, with the ns-laser assisted annealing, crystallization depths of $70 \mathrm{~nm}, 120 \mathrm{~nm}$ and 700 $\mathrm{nm}$ were estimated at $355 \mathrm{~nm}, 532 \mathrm{~nm}$ and $1064 \mathrm{~nm}$ wavelengths, respectively. The laser fluence required to achieve crystallization is around $210 \mathrm{~mJ} / \mathrm{cm}^{2}$ at $355 \mathrm{~nm}, 250 \mathrm{~mJ} / \mathrm{cm}^{2}$ at $532 \mathrm{~nm}$, and $1200 \mathrm{~mJ} / \mathrm{cm}^{2}$ at $1064 \mathrm{~nm}$. Based on Fig. 9(d), with the ps-laser annealing, crystallization depth of $50 \mathrm{~nm}$ was estimated at $1064 \mathrm{~nm}$ and the laser fluence around 60 $\mathrm{mJ} / \mathrm{cm}^{2}$ is required to achieve the crystallization which is significantly less as compared to the ns-laser annealing.

In the case of the ps-laser annealing, as the free carrier absorption is very large at wavelengths of $355 \mathrm{~nm}$ and $532 \mathrm{~nm}$, the depth of annealing is very much limited to few tens of nm and higher pulse energy leads to an ablation of a film. Figures 10(a) and (b) shows the estimated temperature distribution at the surface and at the interface by the ns- and ps-laser annealing at the wavelength of $1064 \mathrm{~nm}$, when the a-Si film thickness is $400 \mathrm{~nm}$. In the case of the ns-laser assisted annealing, the interface temperature was estimated to be around $1200 \mathrm{~K}$, whereas by with the ps-laser assisted annealing, the interface temperature is expected to be around $400 \mathrm{~K}$. Based on this, the heat diffusion is also quite low with the ps-laser treatment. 

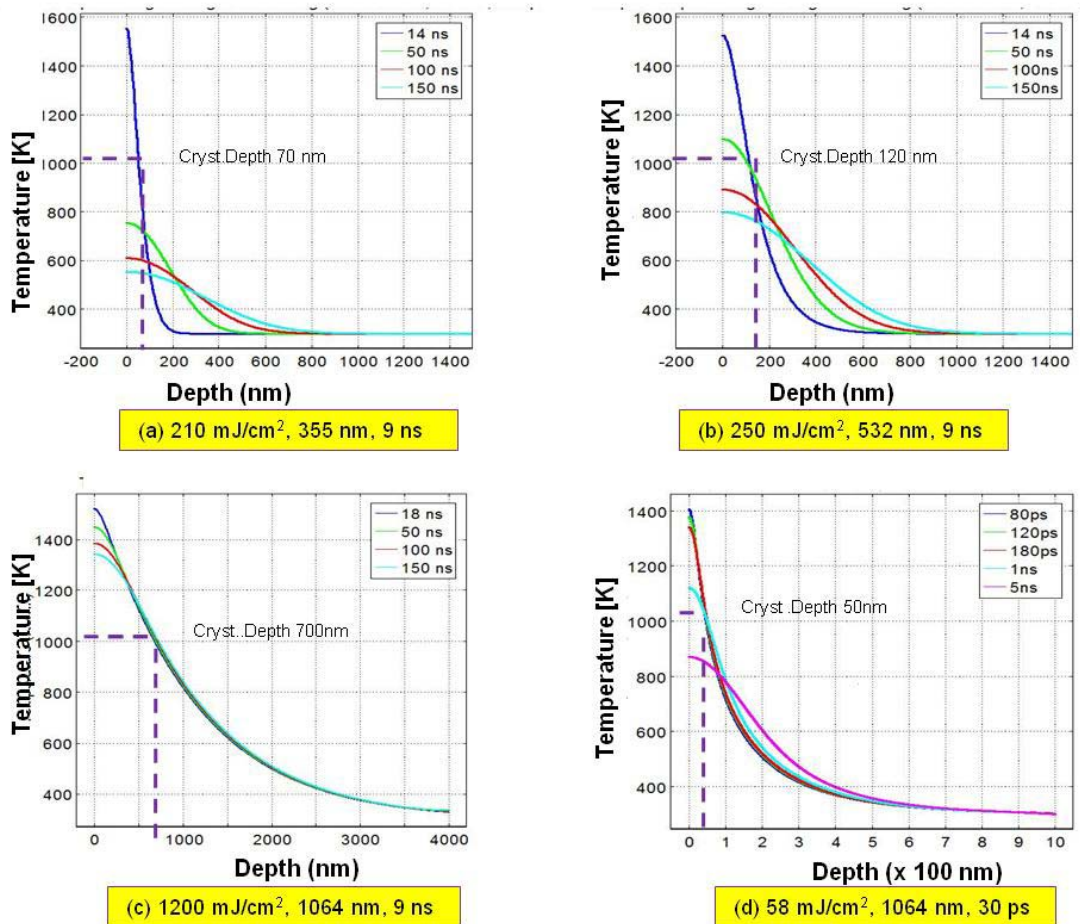

Fig. 9. Temperature distribution along the depth

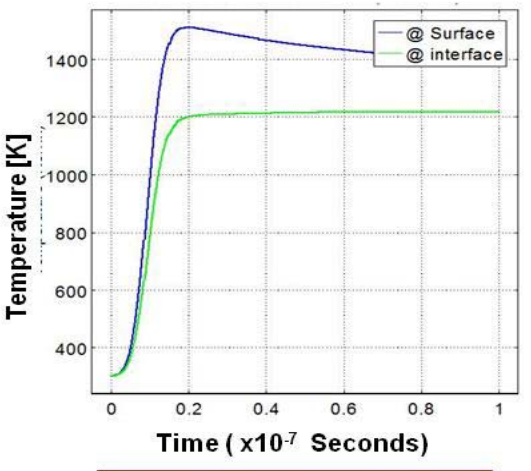

(a) $1200 \mathrm{~m} \mathrm{~J} / \mathrm{cm}^{2}, 1064 \mathrm{~nm}, 9 \mathrm{~ns}$

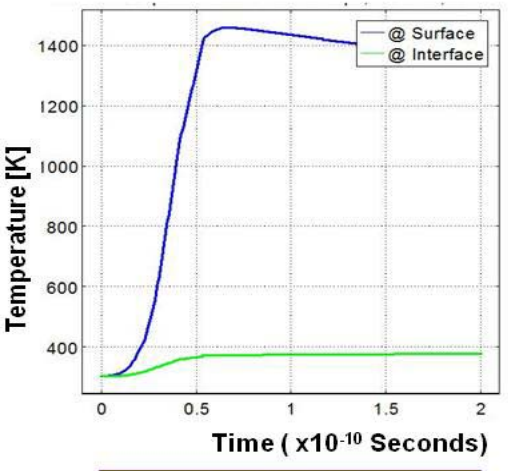

(b) $58 \mathrm{~m} \mathrm{~J} / \mathrm{cm}^{2}, 1064 \mathrm{~nm}, 30 \mathrm{ps}$

Fig. 10. Temperature at surface and at interface by ns- and ps- pulse

\section{Summary}

In summary, based on above study, it is clear that a-Si films deposited on the crystalline silicon wafers can be annealed and simultaneously textured using a ps-laser with a low energy and with a narrow heat affected zone. The crystalline properties obtained with the ps-laser are comparable with that of the ns-laser treated samples. Theoretical modeling results shows that with the ns-laser annealing at a longer wavelength (1064 nm) due to the weak absorption, higher laser fluence is required to raise the film temperature to a required crystallization level. Further, the heat diffusion zone is large resulting in a high a-Si/substrate interface temperature. In the experimental results with ns-laser annealing, crystalline region is observed at $900-1200 \mathrm{~mJ} / \mathrm{cm}^{2}$ which matches with the theoretical 
result. On the other hand, in the case of the ps-laser annealing at the same wavelength requires very less laser fluence. In case of ps-laser annealing, crystalline region is observed at $150-195 \mathrm{~mJ} / \mathrm{cm}^{2}$ experimentally. The required laser fluence for laser annealing is larger than that of the theoretically estimated laser fluence value around $60 \mathrm{~mJ} / \mathrm{cm}^{2}$. Experimentally, the $\mathrm{Rq}$ values of around $150 \mathrm{~nm}$ and $570 \mathrm{~nm}$ are obtained with the ns-laser and the ps-laser assisted texturing, respectively. Although the crystallization characteristics of a-Si films with the ns-laser and the ps-laser are comparable, texturization characteristics in terms of a roughness parameter and heat diffusion characteristics are different. Also, the estimated heat diffusion length with the ps-laser annealing is smaller as compared to that of the ns-laser assisted annealing. Hence for photovoltaic applications, a-Si film annealing and texturing can be considered with the ps-laser at $1064 \mathrm{~nm}$.

\section{Acknowledgements}

Part of the project was supported by the Science \& Engineering Research Board, New Delhi, India (SR/S3/MERC/0085/2010). I.A. Palani is also grateful to the Kyushu University for the Kyushu University Friendship Scholarship. Authors are also grateful to Dr. M. Kumaravel of IIT Madras, Prof. Tatsuo Okada and Prof. Yuji Oki of Kyushu University, Japan for their support and providing the experimental facility.

\section{References}

(1) Aberle, G., Fabrication and characterization of crystalline silicon thin film materials for solar cells, Thin Solid Films, Vol.511 (2006), pp. 26 -34.

(2) Guha, S., Thin films silicon solar cells grown near the edge of amorphous to microcrystalline transition, Solar Energy, Vol.77 (2004), pp. 887-892.

(3) Klinger, D., Lusakowska, E., Kret, S., Kozankiewicz., B. and Zymierska, D., Surface morphology created by nanosecond laser annealing of amorphised Si layer-Investigations by complementary methods, Radiation Physics and Chemistry, Vol.80, No.10 (2011), pp. 1031-1035.

(4) Palani, I. A., Vasa, N. J. and Singaperumal, M., Crystallization and ablation in annealing of amorphous-Si thin film on glass and crystalline-Si substrates by third harmonics of Nd3+:YAG laser, Material Science and Semiconductor Processing, Vol.11 (2008), pp.107-116.

(5) Palani, I. A., Vasa, N. J., Singaperumal, M. and Okada, T., Investigation on laser annealing and subsequent laser-nanotexturing of amorphous silicon films for photovoltaic application, Journal of Laser Micro and Nano Engineering, Vol.5 (2010), pp. 150-155.

(6) Vikram Iyengar,V., Nayak,B.K., More,K.L., Meyer,H.M., Biegalski,M.D., Li,J.V., and Gupta, M.C., Properties of ultrafast laser textured silicon for photovoltaics, Solar Energy Materials and Solar Cells, Vol.95(2011), pp.2745 -2751.

(7) Afonso, C. N., Solis, J., Vega, F. and J. Siegel., Szyszko, W, Solidification phenmomena in Ge.Films upon nano- and pico-second laser pulse melting, Applied Surface Science, Vol.109 (1997), pp. 20-24.

(8) Van vechten,J and Tsu.R., Non Thermal pulsed annealing of Silicon: Plasma annealing, Physics letters, Vol.74A, No.6 (1979), pp.422-426.

(9) Yoffa.E.J., Dynamics of dense laser irradiated plasma, Physical Review B, Vol.21, No.6 (1980), pp.2415-2424.

(10) Guntis, M., Mikhail,C., Jelena,B., Ivars,T., Boris,P., Robert,K., Alekse,M., Georgy,C. and Pavel,B, Structure and characteristics of laser crystallized thin 
amorphous silicon films, Energy Procedia, Vol.3 (2011), pp.42-45.

(11) Azuma,H., Takeuchi,A., Ito,T., Fukushima,H., Motohiro,T. and Yamaguchi, Pulsed KrF excimer laser annealing of silicon solar cell, Solar Energy Materials \& Solar Cells, Vol.74 (2002), pp.289-294.

(12) Zhijun,Y., Qihong,L., Jun,Z., Jingxing,D, Yunrong,W., Zhijiang,W., Hongming, $Z$ and Guohua.W, Numerical and experimental analysis on green laser crystallization of amorphous silicon thin films, Optics \& laser Technology, Vol.41 (2009), pp.380-383.

(13) Von der Linde,D. and Fabricius,N, Observation of electronic plasma in picoseconds laser annealing of silicon, Applied Physics Letters, Vol.41 (1982), pp. 991-993.

(14) Lietoila,A., Gold, R.B., and Gibbons,J.F., Calculation of carrier and lattice temperatures induced in Si by picosecond laser pulses, Applied Physics Letters, Vol.39 (1981), pp.810-816.

(15) Fabijańska, A., F. Kostrubiec, T. Koszmider, R. Pawlak, M. Tomczyk and Walczak, M, Thermal changes of the surface tension in selected laser microtechnologies, Proceedings of International Conference Microtechnology and Thermal Problems in Electronics, (2007), pp.167-172. 